\title{
PHYTOCHEMICAL CONSTITUENT AND SUBCHRONIC TOXICITY EVALUATION OF ETHANOL EXTRACT OF Saurauia vulcani Korth Lour. LEAVES IN WISTAR RATS
}

\author{
Rosidah $^{1}$, S.S. Widjaja ${ }^{2}$, D. Pertiwi ${ }^{3, \bowtie}$ Yuandani $^{1}$, N.J.P. Simanjuntak ${ }^{1}$, \\ and D. Satria ${ }^{4}$ \\ ${ }^{1}$ Department of Pharmacology, Faculty of Pharmacy, Universitas Sumatera Utara, \\ Medan, 20155, Indonesia \\ ${ }^{2}$ Department of Biochemistry, Faculty of Medicine, Universitas Sumatera Utara, \\ Medan, 20155, Indonesia \\ ${ }^{3}$ Department of Pharmaceutical Biology, Faculty of Pharmacy, Universitas Sumatera Utara, \\ Medan, 20155, Indonesia \\ ${ }^{4}$ Department of Pharmaceutical Chemistry, Faculty of Pharmacy, Universitas Sumatera Utara, \\ Medan, 20155, Indonesia \\ ${ }^{\otimes}$ Corresponding Author: dewipertiwi@usu.ac.id
}

\begin{abstract}
The objective of this study is to determine phytochemical constituents and to evaluate the subchronic toxic effect in the ethanol extract of Saurauia vulcani leaves. Phytochemical screening revealed that the extract consists of flavonoids, tannins, glycosides, saponins and steroids/triterpenoids. The extract was orally administered to the rats at doses of $50,250,500,1000 \mathrm{mg} / \mathrm{kg}$ body weight (BW) for 90 days. Signs of toxicity and mortality were observed for 90 days and 118 days. The biochemistry, hematology, macro pathology, relative organ weight and histopathology examination were also performed. The macroscopic and microscopic examinations of liver, lung, kidney, spleen and heart showed normal color and texture after treatment with ethanol extract of Saurauia vulcani leaves at doses of 50, 250,500 and $1000 \mathrm{mg} / \mathrm{kg}$ BW. There was no effect on hematological parameters at all doses of the extract. It can be concluded that ethanol extract of Saurauia vulcani leaf is nontoxic at doses 50, 250, 500 and $1000 \mathrm{mg} / \mathrm{kg} \mathrm{BW}$ for long-term use.
\end{abstract}

Keywords: Subchronic Toxicity, Ethanol Extract Saurauia vulcani, Macroscopic, Microscopic

RASĀYAN J. Chem., Vol. 14, No.3, 2021

\section{INTRODUCTION}

Diabetes is a disorder impaired metabolism of carbohydrates, proteins, and fats due to inadequate or inefficient activity of insulin. It is characterized by fasting hyperglycemia, postprandial hyperglycemia and hyperlipidemia, resulting from defects in carbohydrate, fat and protein metabolism..$^{1-3}$ The World Health Organization estimates that the total number of diabetics worldwide will reach 333 million in 2025 from 135 million in 1995. Diabetes mellitus is an enormous public health problem globally, associated with high morbidity and mortality It is recognized as a wide-reaching chronic disorder affecting almost all age groups. According to a report of IDF 2017, there are 422 million people experiencing diabetes in the world and this figure is expected to rise 629 million in $2045 .{ }^{4}$ There are much research has been performed to manage diabetes and oral hypoglycemic agents have been developed but due to certain limitations of these agents the scientific community is still trying to explore some new oral hypoglycemic agents. ${ }^{5}$

Currently, antidiabetic drugs synthetic hypoglycemic agents such as sulfonylureas group, insulin treatment are given parenterally and specific enzyme inhibitors like acarbose, miglitol are used for patients. However, these drugs are expensive and usually have side effects like insulin resistance, anorexia nervosa, brain atrophy, hepatotoxicity, abdominal pain, and flatulence ${ }^{6-8}$ For a long time, diabetes has been treated with medicinal plants and the scope of exploration of new antidiabetic drugs via herbs is still of interest. ${ }^{9}$ The use of medicinal plants is increasing because of the fewer side effects compared to synthetic drugs. ${ }^{10}$ 
RASĀYAN J. Chem.

Vol. 14 | No. 3 |1441-1446| July - September | 2021

Scientific data have indicated that medicinal plant and their products possess antidiabetic properties with less toxicity and side-effect. ${ }^{11}$

A natural product that has antihyperglycemic activity and has the potential to be developed into antidiabetic is pirdot (Saurauia vulcani Korth). Pirdot is an endemic plant that is widely found in Sumatera Utara. Ethanol extract of pirdot leaves at a dose of $200 \mathrm{mg} / \mathrm{kg}$ BW has activity in reducing blood glucose levels on glucose tolerance test in mice. ${ }^{12,13}$ In research showed that Saurauia Roxburghii has antioxidant and antidiabetic effects. ${ }^{14}$

\section{Plant and Chemicals Material}

\section{EXPERIMENTAL}

Fresh leaves of Saurauia vulcani Korth were collected from Kabanjahe district, Karo subdistrict, Sumatera Utara province, Indonesia. Chemicals used were distilled water, Na CMC, ethanol 96\%.

\section{Preparation of Extracts}

The air-dried and powdered leaves of Saurauia vulcani Korth (500 g) were macerated with ethanol 96\% $(3 \times 3 \mathrm{~d}, 7.5 \mathrm{~L})$. The filtrate was collected and then evaporated to give a viscous fraction and then freezedried to dry.

\section{Animals}

Male and female Wistar rats in age 6-8 week old, weighing 150-200 g were used. The animals were acclimatized for 7-14 days in the experiment room.

\section{Phytochemical Screening}

Phytochemical screening was conducted using a standard method for detection of alkaloids, flavonoids, tannins, glycosides, saponins and steroids/triterpenoids. ${ }^{15}$

\section{Subchronic Toxicity Study}

The subchronic toxicity evaluation followed OECD guidelines ${ }^{16}$ There were 7 groups of the animal consists of 5 females and males rats, a negative control was given Na-CMC $0.5 \%$, positive control was given Saurauia vulcani ethanol extract 50, 250,500, and $1000 \mathrm{mg} / \mathrm{kg} \mathrm{BW}$, and satellite groups was given Na CMC $0.5 \%$ and Saurauia vulcani ethanol extract $1000 \mathrm{mg} / \mathrm{kg} \mathrm{BW}$ with extended observations. The treatments were given orally at a single dose to the rats for 90 days. The body weight, toxic symptoms and mortality were observed for 90 days each week. For the satellite groups, further observations were carried out for 118 days aimed at evaluating the recovery process from the toxic effect that occurred.

\section{Analysis of Hematological and Biochemistry}

Blood for hematological and biochemical clinical parameters was collected by the tail, arterial and cardiac arteries of rats. Plasma was used in hematological, and serum was used to determine ALT, AST, creatinine, and BUN levels.

\section{Macroscopic Assessment of the Organs}

The organs were taken from rats namely liver, spleen, kidney, lung, and heart) then washed to observe the color, surface, and consistency. After that, the organ was dried and weighed to determine the relative organ weights which are calculated using the following formula:

$$
\text { Weight Relative }=[\text { Weight of Organ }] /[\text { Weight of Animal }]
$$

\section{Microscopic Study of the Organs}

The rat's organs were immersed in a $10 \%$ formalin buffer solution and then implanted in paraffin. The microtome was used to slice then stained with hematoxylin and eosin. Then, the organ slices were observed using a microscope.

\section{Statistical Analysis}

Data were analyzed with one-way analysis of variance (ANOVA) and Post Hoc Tuckey to see differences with significance $(p>0.05)$ between test groups. 
RASĀYAN J. Chem.

Vol. 14 | No. 3 |1441-1446| July - September | 2021

\section{Phytochemical Constituent}

\section{RESULTS AND DISCUSSION}

The ethanol extract of Saurauia vulcani contained flavonoids, glikocyde, saponins, tannins and steroids/ triterpenoids in phytochemical screening.

\section{Signs of Toxicity}

The observation was made for 90 and 118 days for the signs of toxicity such as salivation, seizure, changes of eye mucosa, diarrhea, changes of fur and skin, walking with the abdomen and walking backward. Clinical signs were evaluated qualitatively (presence or absence of the clinical signs). The clinical signs appeared after treatment by extract for 90 days. Based on the results of qualitative observations, there were no signs of toxicity coming out of all groups, both male and female animals. The activities and habits of the test animals are normal, there is no evidence of hypersalivation, diarrhea, convulsions, or signs of pain.

\section{Body Weight Observation}

There were none of the animals tested suffered weight loss in this study. All the treatment and control groups displayed weight gain, so did the females in the control group. The ethanol extract of Saurauia vulcani not increased the body weight and did not differ significantly $(p>0.05)$ with the normal group. Some specific indicators for toxicity are clinical symptoms and weight. Every day, animals clinical symptoms were observed and weight was measured regularly. Rapid and significant weight loss is usually a sign of an unhealthy body. Weight loss can be caused by not consuming enough food and drink, signs of a toxic or specific disease. ${ }^{17}$

\section{Mortality}

There are no rats died among treatment.

\section{Macroscopic and Microscopic Examination}

At the end of the experiment over 90 days and 118 days, macropathology and histopathology were observed. The liver and kidney of rats were observed. The microscopic evaluation of the liver on treatment groups at the dose of 50 and $250 \mathrm{mg} / \mathrm{kg} \mathrm{BW}$ was comparable with control groups. Whereas, at the dose of $500 \mathrm{mg} / \mathrm{kg}$ $\mathrm{BW}$ there was sinusoidal dilatation and hydrotropic degeneration, at dose $1000 \mathrm{of} \mathrm{mg} / \mathrm{kg} \mathrm{BW}$ there was necrosis of hepatocyte. This condition was reversible at a satellite dose of $1000 \mathrm{mg} / \mathrm{kg} \mathrm{BW}$ (Fig.-1 and Fig.2).

The organs observed were Liver (A) and Kidney (B) on; (1) normal group; (2) extract group with a dose of $50 \mathrm{mg} / \mathrm{kg} \mathrm{BW}$; (3) extract group with a dose of $250 \mathrm{mg} / \mathrm{kg} \mathrm{BW}$; (4) extract group with a dose of $500 \mathrm{mg} / \mathrm{kg}$ BW; (5) the extract group with a dose of $1000 \mathrm{mg} / \mathrm{kg} \mathrm{BW}$.

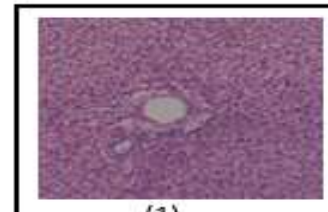

(1)

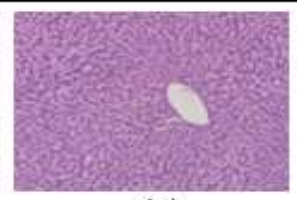

(2)

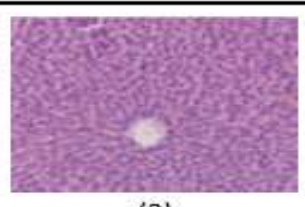

(3)

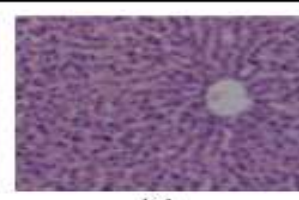

(4)

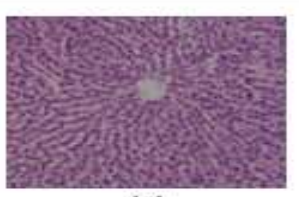

(5)

(A)

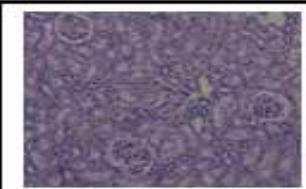

(1)

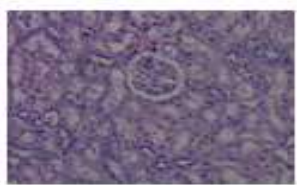

(2)

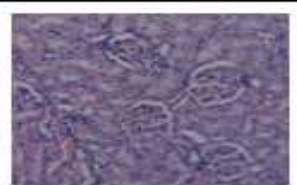

(3)

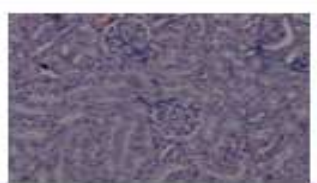

(4)

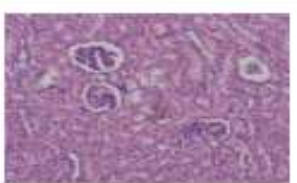

(5)

(B)

Fig.-1: Representative of microscopic changes after treatment with Saurauia vulcani Leaf Extract at doses of 50, 250, 500 and $1000 \mathrm{mg} / \mathrm{kg} \mathrm{BW}$ in male rats $(10 \times 10)$, scale bars: $10 \times 40$. 
RASĀYAN J. Chem.

Vol. 14 | No. 3 |1441-1446| July - September | 2021

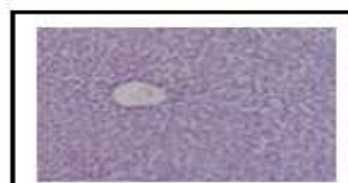

(1)

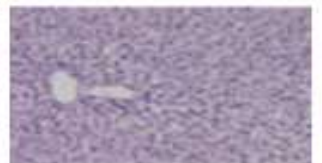

(2)

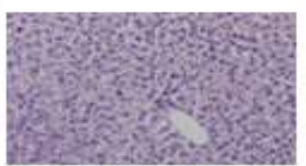

(3)

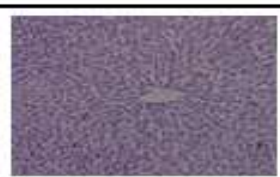

(4)

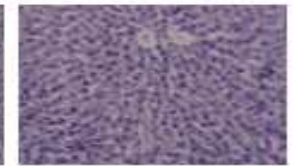

(5)

(A)

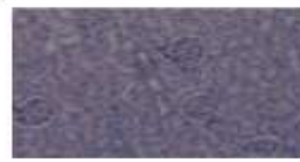

(1)

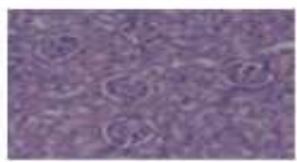

(2)

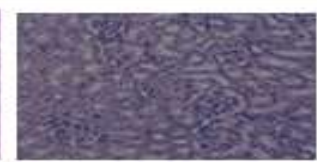

(3)

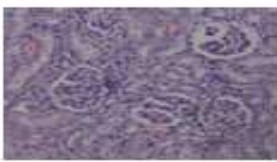

(4)

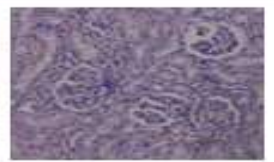

(5)

(B)

Fig.-2: Representative of microscopic changes after treatment with Saurauia vulcani Leaf Extract at doses of 50, 250,500 and $1000 \mathrm{mg} / \mathrm{kg} \mathrm{BW}$ in female rats $(10 \times 10)$, scale bars: $10 \times 40$.

\section{Relative Organ Index}

The effect of Saurauia vulcani leaf extract on male wistar rats relative organ index did not statistically significant with the normal group $(p>0.05)$, so did female wistar rats. There was weight of the liver, kidney, lung, heart, and spleen was measured.

\section{Biochemistry Parameters}

The statistical analysis using one-way ANOVA showed that there was no significant difference $(p>0.05)$ (Table-1 and Table-2).

Table-4: Effect of Saurauia vulcani Leaf Extract on Hematological Parameters on Male Wistar Rats (Mean \pm SD)

\begin{tabular}{|c|c|c|c|c|c|c|c|}
\hline \multirow[b]{2}{*}{$\begin{array}{c}\text { Profiles of } \\
\text { blood (Mean } \pm \\
\text { SD) }\end{array}$} & \multicolumn{7}{|c|}{ Group } \\
\hline & $\begin{array}{c}\text { CMC Na } \\
0,5 \%\end{array}$ & $\begin{array}{l}\text { EESL } 50 \\
\mathrm{mg} / \mathrm{kg} \text { bw }\end{array}$ & $\begin{array}{c}\text { EESL } \\
250 \\
\mathrm{mg} / \mathrm{kg} \\
\text { bw }\end{array}$ & $\begin{array}{c}\text { EESL } \\
500 \\
\mathrm{mg} / \mathrm{kg} \\
\mathrm{bw}\end{array}$ & $\begin{array}{l}\text { EESL } \\
1000 \\
\mathrm{mg} / \mathrm{kg} \\
\text { bw }\end{array}$ & $\begin{array}{c}\text { Satellite } \\
\text { CMC Na } \\
0,5 \%\end{array}$ & $\begin{array}{c}\text { Satellite } \\
\text { EESL } \\
1000 \\
\mathrm{mg} / \mathrm{kg} \mathrm{bw}\end{array}$ \\
\hline WBC & $\begin{array}{c}7.04 \pm \\
1.15 \\
\end{array}$ & $\begin{array}{c}7.66 \pm \\
1.29 \\
\end{array}$ & $\begin{array}{c}8.32 \pm \\
0.91 \\
\end{array}$ & $\begin{array}{c}8.43 \pm \\
0.67 \\
\end{array}$ & $\begin{array}{c}9.14 \pm \\
1.61 \\
\end{array}$ & $\begin{array}{c}7.06 \pm \\
1.47 \\
\end{array}$ & $\begin{array}{c}9.18 \pm \\
0.98 \\
\end{array}$ \\
\hline $\mathrm{RBC}$ & $\begin{array}{c}8.37 \pm \\
1.09 \\
\end{array}$ & $\begin{array}{c}8.87 \pm \\
0.94 \\
\end{array}$ & $\begin{array}{c}8.35 \pm \\
0.97 \\
\end{array}$ & $\begin{array}{c}7.95 \pm \\
1.30 \\
\end{array}$ & $\begin{array}{c}7.12 \pm \\
1.45 \\
\end{array}$ & $\begin{array}{c}8.21 \pm \\
1.20 \\
\end{array}$ & $\begin{array}{c}7.03 \pm \\
0.57\end{array}$ \\
\hline Platelet & $\begin{array}{c}881.60 \pm \\
146.64 \\
\end{array}$ & $\begin{array}{c}899.40 \pm \\
167.51 \\
\end{array}$ & $\begin{array}{c}892.40 \pm \\
167.17 \\
\end{array}$ & $\begin{array}{c}905.80 \pm \\
120.01 \\
\end{array}$ & $\begin{array}{c}943.40 \pm \\
161.13 \\
\end{array}$ & $\begin{array}{c}895.60 \pm \\
106.66 \\
\end{array}$ & $\begin{array}{c}967.00 \pm \\
102.71 \\
\end{array}$ \\
\hline Hemoglobin & $\begin{array}{c}15.71 \pm \\
1.28 \\
\end{array}$ & $\begin{array}{c}15.31 \pm \\
0.86\end{array}$ & $\begin{array}{l}15.56 \\
\pm 1.15 \\
\end{array}$ & $\begin{array}{c}14.60 \pm \\
0.78\end{array}$ & $\begin{array}{c}14.74 \pm \\
1.29 \\
\end{array}$ & $\begin{array}{c}15.12 \pm \\
0.90 \\
\end{array}$ & $\begin{array}{c}16.69 \pm \\
1.96 \\
\end{array}$ \\
\hline Hematocrit & $\begin{array}{c}48.11 \pm \\
4.53\end{array}$ & $\begin{array}{c}44.92 \pm \\
4.18 \\
\end{array}$ & $\begin{array}{c}46.60 \pm \\
4.19 \\
\end{array}$ & $\begin{array}{c}48.53 \pm \\
4.67 \\
\end{array}$ & $\begin{array}{c}47.67 \pm \\
4.83 \\
\end{array}$ & $\begin{array}{c}46.48 \pm \\
3.00\end{array}$ & $\begin{array}{c}51.79 \pm \\
2.36 \\
\end{array}$ \\
\hline $\mathrm{MCH}$ & $\begin{array}{c}17.20 \pm \\
0.86 \\
\end{array}$ & $\begin{array}{c}17.98 \pm \\
1.04 \\
\end{array}$ & $\begin{array}{c}18.22 \pm \\
1.24 \\
\end{array}$ & $\begin{array}{c}18.10 \pm \\
0.94 \\
\end{array}$ & $\begin{array}{c}17.60 \pm \\
1.34 \\
\end{array}$ & $\begin{array}{c}17.32 \pm \\
0.77 \\
\end{array}$ & $\begin{array}{c}17.42 \pm \\
0.90 \\
\end{array}$ \\
\hline MCV & $\begin{array}{c}54.60 \pm \\
1.41\end{array}$ & $\begin{array}{c}54.48 \pm \\
2.57\end{array}$ & $\begin{array}{c}54.12 \pm \\
2.53\end{array}$ & $\begin{array}{c}55.26 \pm \\
1.56\end{array}$ & $\begin{array}{c}54.38 \pm \\
2.53\end{array}$ & $\begin{array}{c}54.82 \pm \\
0.85\end{array}$ & $\begin{array}{c}52.74 \pm \\
0.60\end{array}$ \\
\hline $\mathrm{MCHC}$ & $\begin{array}{c}32.74 \pm \\
1.16 \\
\end{array}$ & $\begin{array}{c}34.52 \pm \\
2.12 \\
\end{array}$ & $\begin{array}{c}34.68 \pm \\
2.26 \\
\end{array}$ & $\begin{array}{c}35.86 \pm \\
2.05 \\
\end{array}$ & $\begin{array}{c}35.36 \pm \\
2.35 \\
\end{array}$ & $\begin{array}{c}33.04 \pm \\
1.12 \\
\end{array}$ & $\begin{array}{c}34.72 \pm \\
2.52 \\
\end{array}$ \\
\hline Eosinophil & $\begin{array}{c}3.20 \pm \\
0.71 \\
\end{array}$ & $\begin{array}{c}3.00 \pm \\
0.53 \\
\end{array}$ & $\begin{array}{c}3.04 \pm \\
0.64 \\
\end{array}$ & $\begin{array}{c}3.08 \pm \\
0.55 \\
\end{array}$ & $\begin{array}{c}3.16 \pm \\
0.68 \\
\end{array}$ & $\begin{array}{c}3.08 \pm \\
0.60 \\
\end{array}$ & $\begin{array}{c}3.18 \pm \\
0.56 \\
\end{array}$ \\
\hline Monocyte & $\begin{array}{c}1.80 \pm \\
0.53 \\
\end{array}$ & $\begin{array}{c}2.76 \pm \\
0.23 \\
\end{array}$ & $\begin{array}{c}2.16 \pm \\
0.50 \\
\end{array}$ & $\begin{array}{c}2.28 \pm \\
0.65\end{array}$ & $\begin{array}{c}2.32 \pm \\
0.66 \\
\end{array}$ & $\begin{array}{c}2.00 \pm \\
0.43 \\
\end{array}$ & $\begin{array}{c}2.48 \pm \\
0.54 \\
\end{array}$ \\
\hline Basophil & $\begin{array}{c}0.38 \pm \\
0.09\end{array}$ & $\begin{array}{c}0.35 \pm \\
0.09\end{array}$ & $\begin{array}{c}0.35 \pm \\
0.08\end{array}$ & $\begin{array}{c}0.36 \pm \\
0.10\end{array}$ & $\begin{array}{c}0.41 \pm \\
0.09\end{array}$ & $\begin{array}{c}0.38 \pm \\
0.05\end{array}$ & $\begin{array}{c}0.41 \pm \\
0.06\end{array}$ \\
\hline
\end{tabular}


RASĀYAN J. Chem.

Vol. 14 | No. 3 |1441-1446| July - September | 2021

\begin{tabular}{c|c|c|c|c|c|c|c}
\hline \multirow{2}{*}{ Lymphocyte } & $64.54 \pm$ & $67.10 \pm$ & $71.38 \pm$ & $69.08 \pm$ & $66.86 \pm$ & $63.48 \pm$ & $68.14 \pm$ \\
& 15.18 & 14.21 & 10.51 & 14.66 & 10.32 & 4.27 & 7.41 \\
\hline & $29.28 \pm$ & $27.34 \pm$ & $31.22 \pm$ & $31.08 \pm$ & $33.52 \pm$ & $29.86 \pm$ & $32.96 \pm$ \\
Neutrophil & 6.75 & 6.67 & 5.93 & 7.21 & 9.44 & 1.58 & 5.14 \\
\hline
\end{tabular}

Table-5: Effect of Saurauia vulcani Leaf Extract on Hematological Parameters on Female Rats (Mean \pm SD)

\begin{tabular}{|c|c|c|c|c|c|c|c|}
\hline \multirow[b]{2}{*}{$\begin{array}{c}\text { Profiles of } \\
\text { blood (Mean } \pm \\
\text { SD) }\end{array}$} & \multicolumn{7}{|c|}{ Group } \\
\hline & $\begin{array}{c}\mathrm{CMC} \mathrm{Na} \\
0,5 \%\end{array}$ & $\begin{array}{c}\text { EESL } \\
50 \\
\mathrm{mg} / \mathrm{kg} \\
\mathrm{bw}\end{array}$ & $\begin{array}{c}\text { EESL } \\
250 \\
\mathrm{mg} / \mathrm{kg} \\
\mathrm{bw}\end{array}$ & $\begin{array}{c}\text { EESL } \\
500 \\
\mathrm{mg} / \mathrm{kg} \\
\mathrm{bw}\end{array}$ & $\begin{array}{l}\text { EESL } \\
1000 \\
\mathrm{mg} / \mathrm{kg} \\
\text { bw }\end{array}$ & $\begin{array}{c}\text { Satellit } \\
\text { CMC Na } \\
0,5 \%\end{array}$ & $\begin{array}{c}\text { Satellit EESL } \\
1000 \mathrm{mg} / \mathrm{kg} \\
\text { bw }\end{array}$ \\
\hline WBC & $\begin{array}{c}6.82 \pm \\
0.53\end{array}$ & $\begin{array}{c}6.85 \pm \\
1.18\end{array}$ & $\begin{array}{c}7.78 \pm \\
1.05\end{array}$ & $\begin{array}{c}8.14 \pm \\
0.71\end{array}$ & $\begin{array}{c}8.92 \pm \\
1.38\end{array}$ & $\begin{array}{c}6.90 \pm \\
1.37\end{array}$ & $\begin{array}{c}8.94 \pm \\
1.25\end{array}$ \\
\hline $\mathrm{RBC}$ & $\begin{array}{c}8.13 \pm \\
0.68\end{array}$ & $\begin{array}{c}8.23 \pm \\
0.91\end{array}$ & $\begin{array}{c}7.46 \pm \\
0.46\end{array}$ & $\begin{array}{c}7.57 \pm \\
0.80\end{array}$ & $\begin{array}{c}6.89 \pm \\
0.68\end{array}$ & $\begin{array}{c}7.78 \pm \\
0.92\end{array}$ & $\begin{array}{c}6.69 \pm \\
0.93\end{array}$ \\
\hline Platelet & $\begin{array}{c}789.60 \pm \\
128.50\end{array}$ & $\begin{array}{c}839.00 \pm \\
198.49\end{array}$ & $\begin{array}{c}912.40 \pm \\
195.04\end{array}$ & $\begin{array}{c}878.00 \pm \\
123.14\end{array}$ & $\begin{array}{c}795.00 \pm \\
210.86\end{array}$ & $\begin{array}{c}884.00 \pm \\
94.15\end{array}$ & $\begin{array}{c}806.20 \pm \\
167.99\end{array}$ \\
\hline Hemoglobin & $\begin{array}{c}15.56 \pm \\
0.97\end{array}$ & $\begin{array}{c}25.75 \pm \\
1.25\end{array}$ & $\begin{array}{c}15.09 \pm \\
1.09\end{array}$ & $\begin{array}{c}15.62 \pm \\
1.45\end{array}$ & $\begin{array}{c}15.18 \pm \\
1.03\end{array}$ & $\begin{array}{c}15.75 \pm \\
1.45\end{array}$ & $\begin{array}{c}15.26 \pm \\
0.19\end{array}$ \\
\hline Hematocrit & $\begin{array}{c}45.00 \pm \\
4.70\end{array}$ & $\begin{array}{c}46.84 \pm \\
7.18 \\
\end{array}$ & $\begin{array}{c}45.66 \pm \\
5.64\end{array}$ & $\begin{array}{c}47.32 \pm \\
6.17\end{array}$ & $\begin{array}{c}45.31 \pm \\
6.00\end{array}$ & $\begin{array}{c}46.48 \pm \\
6.15\end{array}$ & $\begin{array}{c}46.25 \pm \\
6.05\end{array}$ \\
\hline $\mathrm{MCH}$ & $\begin{array}{c}18.30 \pm \\
1.71\end{array}$ & $\begin{array}{c}18.66 \pm \\
1.60\end{array}$ & $\begin{array}{c}18.16 \pm \\
1.86\end{array}$ & $\begin{array}{c}18.62 \pm \\
1.67\end{array}$ & $\begin{array}{c}18.36 \pm \\
1.90\end{array}$ & $\begin{array}{c}18.64 \pm \\
1.45\end{array}$ & $\begin{array}{c}18.90 \pm \\
1.57\end{array}$ \\
\hline $\mathrm{MCV}$ & $\begin{array}{c}54.12 \pm \\
2.98\end{array}$ & $\begin{array}{c}55.37 \pm \\
3.89\end{array}$ & $\begin{array}{c}56.61 \pm \\
1.04\end{array}$ & $\begin{array}{c}55.93 \pm \\
1.57\end{array}$ & $\begin{array}{c}56.45 \pm \\
1.69\end{array}$ & $\begin{array}{c}54.18 \pm \\
1.74\end{array}$ & $\begin{array}{c}54.74 \pm \\
2.07\end{array}$ \\
\hline $\mathrm{MCHC}$ & $\begin{array}{c}33.72 \pm \\
2.34\end{array}$ & $\begin{array}{c}35.14 \pm \\
2.95\end{array}$ & $\begin{array}{c}36.00 \pm \\
2.79\end{array}$ & $\begin{array}{c}35.56 \pm \\
2.74\end{array}$ & $\begin{array}{c}35.54 \pm \\
2.93\end{array}$ & $\begin{array}{c}35.18 \pm \\
2.09\end{array}$ & $\begin{array}{c}35.88 \pm \\
2.96\end{array}$ \\
\hline Eosinophil & $\begin{array}{c}3.50 \pm \\
0.71\end{array}$ & $\begin{array}{c}3.30 \pm \\
0.80\end{array}$ & $\begin{array}{c}2.86 \pm \\
0.84\end{array}$ & $\begin{array}{c}3.22 \pm \\
0.88\end{array}$ & $\begin{array}{c}3.22 \pm \\
0.72\end{array}$ & $\begin{array}{c}3.24 \pm \\
0.67\end{array}$ & $3.30 \pm 0.62$ \\
\hline Monocyte & $\begin{array}{c}2.00 \pm \\
0.57\end{array}$ & $\begin{array}{c}2.28 \pm \\
0.71\end{array}$ & $\begin{array}{c}2.24 \pm \\
0.76\end{array}$ & $\begin{array}{c}1.82 \pm \\
0.52\end{array}$ & $\begin{array}{c}2.40 \pm \\
0.87\end{array}$ & $\begin{array}{c}2.08 \pm \\
0.64\end{array}$ & $\begin{array}{c}2.56 \pm \\
0.50\end{array}$ \\
\hline Basophil & $\begin{array}{c}0.31 \pm \\
0.07\end{array}$ & $\begin{array}{c}0.36 \pm \\
0.10\end{array}$ & $\begin{array}{c}0.35 \pm \\
0.76\end{array}$ & $\begin{array}{c}0.34 \pm \\
0.09\end{array}$ & $\begin{array}{c}0.36 \pm \\
1.21\end{array}$ & $\begin{array}{c}0.35 \pm \\
0.72\end{array}$ & $\begin{array}{c}0.40 \pm \\
0.06\end{array}$ \\
\hline Lymphocyte & $\begin{array}{c}58.98 \pm \\
16.16\end{array}$ & $\begin{array}{c}60.62 \pm \\
12.06\end{array}$ & $\begin{array}{c}66.52 \pm \\
11.79\end{array}$ & $\begin{array}{c}67.36 \pm \\
14.73\end{array}$ & $\begin{array}{c}72.34 \pm \\
8.38\end{array}$ & $\begin{array}{c}62.38 \pm \\
9.89\end{array}$ & $\begin{array}{c}65.16 \pm \\
9.24\end{array}$ \\
\hline Neutrophil & $\begin{array}{c}27.1 \pm \\
6.34\end{array}$ & $\begin{array}{c}25.92 \pm \\
4.84\end{array}$ & $\begin{array}{c}26.10 \pm \\
5.90\end{array}$ & $\begin{array}{c}29.04 \pm \\
5.45\end{array}$ & $\begin{array}{c}31.80 \pm \\
2.68\end{array}$ & $\begin{array}{c}27.48 \pm \\
3.18\end{array}$ & $\begin{array}{c}31.72 \pm \\
1.80\end{array}$ \\
\hline
\end{tabular}

The histopathological and biochemical parameters showed the effect on the liver, lung, kidney, and heart. There was nota significant difference in ALT, AST, creatinine and BUN levels between the control and extract groups for all doses 50, 250, 500 and $1000 \mathrm{mg} / \mathrm{kg} \mathrm{BW}$. From the result known that the effect of ethanol extract of Saurauia vulcani leaf was not affected on biochemistry parameters. In a previous study, it was concluded that the duration and intensity of exposure to toxic substances also can affect the form and toxicity of a particular substance. ${ }^{18}$ If adaptive biochemical responses are sustained, it can cause pathological and biochemical change or disorder. ${ }^{19}$

\section{CONCLUSION}

The ethanol extract of Saurauia vulcani leaves did not cause a toxic effect in long-term treatment at the dose of 50, 250,500 and $1000 \mathrm{mg} / \mathrm{kg} \mathrm{BW}$. The result of macroscopic and microscopic, there were no changes observed at the dose of $50,250,500$ and $1000 \mathrm{mg} / \mathrm{kg}$ BW compared to the normal group. In addition, the hematological and biochemistry parameters were still within the normal range. And there was no mortality along with treatment.

\section{ACKNOWLEDGEMENT}

The authors are thankful to Rector Universitas Sumatera Utara, for the research funding provided through TALENTA (Penelitian Dasar 2019). 
RASĀYAN J. Chem.

Vol. 14 | No. 3 |1441-1446| July - September | 2021

\section{REFERENCES}

1. American Diabetes Association (ADA), Diagnosis and Classification of Diabetes Mellitus, Diabetes Care, 37, 81(2014), http://doi.org/10.2337/dc14-S081

2. M. R. Taskinen, Atherosclerosis Supplements, 3(1), 47(2002), http://doi.org/10.1016/s1567$\underline{5688(01) 00006-\mathrm{x}}$

3. A. Ceriello, Diabetes, 54, 1(2005), http://doi.org/10.2337/diabetes.54.1.1

4. N. H. Cho, J. E. Shaw, S. Karuranga, Y. Huang, J. D. de Rocha Fernandes, A.W. Ohlrogge, B. Malanda, Diabetes Research and Clinical Practice, 138, 271(2018), http://doi.org/10.1016/j.diabres.2018.02.023

5. G. B. Kavishankar, N. Lakshmidevi, S.M. Murthy, H.S. Prakash, S.R. Niranjana, Journal of Pharmaceutical and Biomedical Sciences, 2, 65(2011).

6. G. Piedrola, E. Novo, F. Escobar, R. Garcia-Robles, The Annales d'Endocrinologie, 62, 7(2001)

7. J. A. Y. Tobias, A. Pinto, F. Neziroglu, International Journal of Eating Disorders, 30, 350(2001), http://doi.org/10.1002/eat.1096

8. R. F. Conniff, J. A. Shapiro, T. B. Seaton, G.A. Bray, The American Journal of Medicine, 98, 443(1995), http://doi.org/10.1016/S0002-9343(99)80343-X

9. R. K. Gupta, A. N. Kesari, P. S. Murthy, R. Chandra, V. Tandon, G. Watal, The Journal of Ethnopharmacology. 99, 75(2005), http://doi.org/10.1016/j.jep.2005.01.048

10. P. C. Situmorang, S. Ilyas, S. Hutahaean, Rosidah and R. D. Manurung, Rasayan Journal of Chemistry, 13(2), 780(2020), http://doi.org/10.31788/RJC.2020.1325621

11. P. George, Journal of Applied Pharmaceutical Science. 1(6), 40(2011).

12. P. Sitorus, Rosidah, D. Satria, Asian Journal of Pharmaceutical and Clinical Research, 11(1), 35(2018), http://doi.org/10.22159/ajpcr.2018.v11s1.26561

13. N. Sormin, P. Sitorus, T. S Matheus, Thesis, Faculty of Pharmacy Universitas Sumatera Utara, Medan, (2014).

14. F. Islam, M. S. Rahman, R. Akter, M. U. Bakar, A. M. Abdullah, N. U. Ahmed, Pharma Science Monitor, 3(4), 2601(2012).

15. J. B. Harborne, Phytochemical Method, Chapman and Hall Ltd, London, p.5 (1973)

16. OECD, Repeated Dose 90-day Oral Toxicity Study in Rodents TG 408, OECD, Paris, p.1 (2018)

17. D. Gupta, S. Bhardwaj, The International Journal of Advanced Research in Pharmaceutical and Bio Sciences, 2(2), 103(2012).

18. U. S. Hastuti, Jurnal Kedokteran Brawijaya, 22(3), 121(2006), http://doi.org/10.21776/ub.jkb.2006.022.03.6

19. J. D. Blachley, J. H. Johnson, J. P. Knochel, The American Journal of the Medical Sciences, 289(1), 22(1985), http://doi.org/10.1097/00000441-198501000-00004

[RJC-6135/2020] 